\title{
In situ bioconversion of coconut oil via coconut solid state fermentation by Geotrichum candidum ATCC 34614
}

\begin{abstract}
Coconut base solid state fermentation was carried out by Geotrichum candidum ATCC 34614 for in situ coconut oil bioconversion. Coconut oil, which contains highly saturated medium chain triglycerides, was partially bioconverted into a combination of medium chain diglycerides, medium chain monoglycerides and medium chain fatty acids by this fungus lipolytic activity. The product demonstrated improved aroma, flavor, thermal behavior and antibacterial activity. Maximum triglycerides conversion (76.5\%) occurred at $40 \%$ moisture content and $50 \%$ oil content after 25 days of incubation. Bioconverted coconut oil revealed as much as $95 \%$ antibacterial activity as well as altered thermal characteristic towards lower melting and higher crystallization points. The fermented culture also revealed highly fruity and flora notes which contained five main short- and medium-chain esters known as aromatic compounds. The present study established the possibility of using G. candidum ATCC 34614 in coconut solid culture for bioconversion of coconut oil, which improves the fermented product characteristics.
\end{abstract}

Keyword: Antibacterial activity; Coconut oil; Geotrichum candidum; In situ bioconversion; Lipolytic activity; Solid state fermentation 